\title{
ON THE QUINTIC SCROLL HAVING THREE DOUBLE CONICS.
}

BY DR. VIRGIL SNYDER.

(Read before the American Mathematical Society, October 25, 1902.)

In his paper on quintic scrolls, * Professor Schwarz mentioned that such a scroll exists having three double conics (type IX of those of genus 0 ). It is now proposed to derive the equation of this surface and to study some of its properties.

A scroll having two double conics will in general be of order 8 and genus 1 . In order to generate a quintic scroll the conics must therefore intersect in two points, one of which is a simple self-corresponding point of the $(2,2)$ correspondence between the points of the two conies, and the other must be a double self-corresponding point. Hence every such quintic scroll must be unicursal. Let the equations of the two conics be

$$
\begin{array}{ll}
c_{1}: & z=0, \quad x^{2}-y w=0 ; \\
c_{2}: & x=0, \quad z^{2}-y w=0,
\end{array}
$$

respectively. The points of each may be expressed parametrically thus :

$$
\begin{array}{ll}
c_{1}: & x=\lambda, \quad y=\lambda^{2}, \quad z=0, \quad w=1 ; \\
c_{2}: & x=0, \quad y=\mu^{2}, \quad z=\mu, \quad w=1 .
\end{array}
$$

The equations of a generator which joins the variable point $\lambda$ to the variable point $\mu$ may be written

$$
\mu x+\lambda z-\lambda \mu w=0, \quad \lambda x-y+\mu z=0 .
$$

The line (1) will generate a unicursal quintic scroll having $c_{1}, c_{2}$ for double conics when $\lambda, \mu$ are connected by a relation of the form described. Since the complete double curve of a unicursal quintic is of order 6 it follows that the residual curve is of order 2. Double generators are impossible since the double element in the $(2,2)$ correspondence is self-corresponding.

* Ueber die geradlinigen Flächen fünften Grades, Crelle's Journal, vol. 67. 
Two rectilinear directrices can not exist on a scroll having other nodal curves; hence the residual nodal curve is a conic $c_{3}$. Since a similar correspondence exists between the new conic and each of the given ones, it follows that the three conics must pass through one common point. No scroll can exist having three double conics which intersect in three distinct pairs of points.

\section{$\S$ 1. Three Double Conics Having One Common Point.}

Let $(0,0,0,1)$ be the point common to the three conics.

Let $c_{1}$ intersect $c_{3}$ in $\left(\alpha, \alpha^{2}, 0,1\right)$, and $c_{2}$ intersect $c_{3}$ in $\left(0, \gamma^{2}, \gamma, 1\right)$.

The conic $c_{3}$ lies in the plane

$$
\alpha x-y+\gamma z=0,
$$

and may be expressed as the intersection of (2) and the most general quadric passing through the three given points. The values of $x: y: z: w$ as determined from (1) and (2) are now to be substituted in the equation of the quadric. The result is the desired $(2,2)$ correspondence between $\lambda, \mu$. Finally, the result of eliminating $\lambda, \mu$ between this equation and those of a generator is the desired equation of the surface.

The correspondence is of the form

$$
\lambda^{2}-\mu^{2} \kappa+m \lambda \mu+\gamma(\kappa+1) \mu-\alpha(\kappa+1) \lambda=0 .
$$

It has a double element at $(\infty, \infty)$ and a simple self-corresponding element at $(0,0)$, hence the point common to all three conics cannot be a double point. Similarly, the point $\alpha$ is a double point in the correspondence between $c_{1}$ and $c_{3}$, and $\gamma$ is a double point in the correspondence between $c_{2}$ and $c_{3}$. Since the plane of each double conic must contain one generator it follows that three generators pass through the point $(0,0,0,1)$. The equations of these generators are

$$
\begin{aligned}
& x=0, \quad \kappa y=(\gamma \kappa+\gamma) z \\
& y=0, \quad \alpha x+\gamma z=0 \\
& z=0, \quad y=(\alpha \kappa+\alpha) x .
\end{aligned}
$$

When $\kappa=1$ they all lie in the plane

$$
y=(\alpha \kappa+\alpha) x+(\gamma \kappa+\gamma) z .
$$


The residual curve in the plane of the three generators is a conic $* c_{4}$; it does not pass through the triple point. This is the only simple conic which can lie on the surface.

Moreover, the scroll is contained in a linear complex and has one algebraic asymptotic line of order 8 . If in addition $m=0$, the complex becomes special and the simple conic breaks up into a simple directrix and a generator. Every plane through the directrix will cut four generators from the surface. The directrix does not intersect any of the double conics, hence the three points in which the three generators from $(0,0,0,1)$ meet the conics again are collinear. The scroll can now be generated by a particular $(1,2)$ correspondence between the points of the conic and the straight line.

The equations of the directrix are

$$
w=0, \quad y=2 \alpha x+2 \gamma z,
$$

and the fourth generator in the plane $y=2 \alpha x+2 \gamma z$ is defined by

$$
\gamma x+\alpha z=2 \alpha \gamma .
$$

This generator connects the three points in which the generators through $(0,0,0,1)$ cut the double conics again. The special asymptotic line of order 8 is now degraded into the directrix of the complex.

When a unicursal quintic scroll has a double conic the residual nodal curve is in general a twisted quartic with two apparent double points. In general a straight line and a quartic having two apparent double points will determine a scroll of order 8 by the condition that each generator is to cut the line once and the quartic twice. The straight line is a double line on the surface and the quartic curve is a triple line. When the surface is reducible and one factor is a quintic having the line as simple directrix and the quartic for double curve, the other component is a cubic having the straight line and the quartic as simple directrices.

Professor Schwarz remarks that the same thing happens if the quartic be replaced by two conics which have two points of intersection. But such is not the case. Let $c_{1}, c_{2}$ be the conics and $l$ the line. Let $c_{1}, c_{2}$ intersect in $W \equiv(0,0,0,1)$ and in $Y \equiv(0,1,0,0)$. The scroll determined by the common se-

* Mr. C. H. Sisam has recently proved that a simple conic lies on the general scroll. Its plane does not pass through the triple point. 
cants of two conics and a line is of order 8, less 1 for each point in which the conics intersect, since the pencils $W l, Y l$ belong to the system. Our scroll is therefore of order 6 and genus 1 , having $c_{1}, c_{2}, l$ for double lines. The residual nodal curve is a twisted cubic which is met once by each generator.

If, however, the line $l$ lies in one of the common tangent planes of the two conics the surface will be of order 5, as may be shown as follows: A generator (1) will cut the line

$$
\begin{gathered}
A_{1} x+B_{1} y+C_{1} z+D_{1} w=0, \quad A_{2} x+B_{2} y+C_{2} z+D_{2} w=0 \\
\text { if } \quad \lambda^{2} \mu(A B)+\lambda \mu^{2}(B C)+\cdots+\lambda(C D)+\mu(A D)=0 .
\end{gathered}
$$

This is in general a $(2,2)$ correspondence of genus 1 with two simple self-corresponding elements at the points of intersection of the conics. If the scroll is to be a quintic, one of these points must be a double element. This condition requires either that the coefficients of $\lambda \mu^{2}$ and $\lambda^{2} \mu$ shall vanish, or that the coefficients of $\lambda$ and $\mu$ shall vanish. In the first case the line $l$ lies in the plane $w=0$, and in the second case $l$ lies in the plane $y=0$. But these are the common tangent planes of $c_{1}$ and $c_{2}$ at $W, Y$. It can easily be verified independently that when $\kappa=1$ and $m=0$ the tangent planes of $c_{1}$ and $c_{2}$ in $(0,1,0,0)$, the tangent plane of $c_{1}$ and $c_{3}$ in $\left(0, \alpha^{2}, \alpha, 1\right)$, and the tangent plane of $c_{2}$ and $c_{3}$ in $\left(\gamma, \gamma^{2}, 0,1\right)$ all pass through the simple directrix.

The only case in which a straight line and two conics which intersect in two points can define a cubic scroll is when the $(2,2)$ correspondence is factorable, for such a surface is determined by a $(1,1)$ correspondence between a line and a conic. For example, the conics $c_{1}, c_{2}$ and the line $y=0, x+z=w$ define a reducible $(2,2)$ correspondence between $\lambda, \mu$ and all lie on the same cubic scroll.

The equation of our quintic, obtained by eliminating $\lambda, \mu$ between (1) and (3), may be written

$$
\left|\begin{array}{llll}
z^{2}-\kappa x^{2}-m x z & y+n x & l z-\kappa y & 0 \\
0 & y+n x & \kappa x y+m y z+n z^{2} & l z-\kappa y \\
w & 1 & x & 0 \\
0 & x & z^{2}-y w & x
\end{array}\right|=0
$$

in which $l \equiv \gamma(\kappa+1), n \equiv-a(\kappa+1)$. 
For the equation of the second surface, having a linear directrix, put $\kappa=1, m=0$.

\section{§ 2. Three Double Conics Having Two Common Points.}

Now consider the case in which the third double conic passes through both points of intersection of $c_{1}, c_{2}$. Let

$$
x=p z
$$

be the equation of its plane. The equation of the general conic lying in this plane and passing through $(0,0,0,1)$, $(0,1,0,0)$ may be written

$$
A z^{2}+B z y+C y w+D z w=0,
$$

from which the $(2,2)$ correspondence becomes

$$
\begin{aligned}
B \lambda^{2} \mu(p+1)+C \lambda^{2} p+\left(A+p^{2} C+C\right) \lambda \mu & +p C \mu^{2} \\
& +p D \mu+D \lambda=0 .
\end{aligned}
$$

In order that the correspondence have a double element at one point of intersection of the two conics, and a simple self corresponding element at the other, either $B=0$ or $D=0$. This requires that the third conic must touch the common tangent plane of $c_{1}, c_{2}$ at one of their points of intersection. Let $B=0$. Then the new conic touches the plane $w=0$ at $(0,1,0,0)$.

The reduced form of the correspondence now becomes

$$
\lambda^{2}+\mu^{2}+m^{\prime} \lambda \mu+n^{\prime} \lambda+l^{\prime} \mu=0
$$

and the analytic procedure is identical with that of the preceding case.

The generators which lie in the planes of the three double conics are respectively

$$
\begin{aligned}
& x=0, \quad y+l^{\prime} z=0 ; \\
& y=0, \quad x-p z=0 ; \\
& z=0, \quad y+n^{\prime} x=0 ;
\end{aligned}
$$

since $\kappa=1$ they cannot lie in one plane. The scroll also contains a simple conic which cuts each of the double conics once. The lines which cut three conics related like $c_{1}, c_{2}$ and the simple conic will in general generate a scroll of order 
8. If one scroll be of order 5, the other will be a cubic and not another quintic, as stated by Schwarz.

In this case the surface does not belong to a linear complex; in particular, therefore, when a quintic scroll has three coaxial double conics it cannot have a rectilinear directrix.

\section{§ 3. Two Double Conics Touching Each Other.}

Any two conics which touch each other but which lie in different planes may be expressed by the equations

$$
\begin{array}{ll}
c_{1}^{\prime}: & z=0, \quad y^{2}-x w=0 ; \\
c_{2}^{\prime}: & x=0, \quad y^{2}-w z=0 .
\end{array}
$$

In parametric representation these become

$$
\begin{array}{ll}
c_{1}^{\prime}: & x=1, \quad y=\mu, \quad z=0, \quad w=\mu^{2} ; \\
c_{2}^{\prime}: & x=0, \quad y=\lambda, \quad z=1, \quad w=\lambda^{2} .
\end{array}
$$

The equations of the line joining $\lambda$ to $\mu$ are

$$
\mu x+\lambda z-y=0, \quad \mu^{2} x+\lambda^{2} z-w=0 .
$$

The most general $(2,2)$ relation between $\lambda, \mu$ which will define a quintic scroll is given by

$$
g \lambda^{2}+f \mu^{2}-\lambda \mu(f+g)+h=0 .
$$

The surface has a third double conic $c_{3}^{\prime}$ defined by

$$
y=0, \quad h x z+g x w-f z w=0 .
$$

The equation of the surface may be written

$$
\left|\begin{array}{rrrl}
g & -(f+g) y & h x-f w & 0 \\
0 & g(x+z) & -(f+g) y & h x-f w \\
z & -2 y z & y^{2}-w x & 0 \\
0 & z(x+z) & -2 y z & y^{2}-w x
\end{array}\right|=0 .
$$

The common tangent to $c_{1}^{\prime}, c_{2}^{\prime}$ is a generator. The generator in the plane $y=0$ is $x+z=0$. The curve of section made by the plane $x+z=0$ is

$$
z y^{2}\left[(f+g)\left(y^{2}+w z\right)+2 z(h z-f w)\right]=0 ;
$$


hence the line $y=0, x+z=0$ is a torsal generator and $x+z=0$ is the torsal plane. The simple conic passes through the point of intersection of the three double conics and touches the generator $x=0, y=0$.

If $f+g=0$ the conic will be factorable. The rectilinear directrix is

$$
x+z=0, \quad h z-f w=0 .
$$

If the simple conic be required to intersect $c_{3}^{\prime}$ in a second point, the former must break up.

When the scroll belongs to a linear complex, the complex must be special. A quintic scroll which has three double conics which touch the same line at the same point, does not exist.

Every quintic scroll having three double conics contains either a simple conic or a simple directrix. If in the former case the planes of all four conics pass through the same point, then the plane of the simple conic contains three generators passing through that point.

CorNell UNIVERsity,

October 10, 1902.

\section{SURFACES REFERRED TO THEIR LINES OF LENGTH ZERO.}

BY DR. L. P. EISENHART.

(Read before the American Mathematical Society, October 25, 1902.)

Consider a surface $S$ referred to a general system of parametric lines, $u=$ const., $v=$ const. Denote by $x, y, z$, the cartesian coördinates of a point on $S$ and $X, Y, Z$, the direction cosines of the normal to the surface. As usual, we put

(1) $E=\sum\left(\frac{\partial x}{\partial u}\right)^{2}, \quad F=\sum \frac{\partial x}{\partial u} \frac{\partial x}{\partial v}, \quad G=\sum\left(\frac{\partial x}{\partial v}\right)^{2}$,

and

(2) $D=\sum X \frac{\partial^{2} x}{\partial u^{2}}, \quad D^{\prime}=\sum X \frac{\partial^{2} x}{\partial u \partial v}, \quad D^{\prime \prime}=\sum X \frac{\partial^{2} x}{\partial v^{2}}$ 